\title{
Displasia fibrosa poliostótica - síndrome de McCune-Albright: reporte de un caso
}

Polyostotic fibrous dysplasia - McCune-Albright syndrome: a case report Displasia fibrosa poliostótica - síndrome de McCune-Albright: relato de um caso

\author{
Dres. Tomás Rienzi ${ }^{1}$, Claudio Silveri², Mariana Risso³ ${ }^{3}$ Beatriz Mendoza ${ }^{4}$
}

\section{Resumen}

Introducción: la displasia fibrosa poliostótica es una patología de muy baja prevalencia, por lo que su diagnóstico pasa desapercibido en la mayoría de los casos. Cuando se asocia a endocrinopatías o a lesiones cutáneas hiperpigmentadas corresponde al síndrome de McCune-Albright.

Caso clínico: escolar de 8 años, sexo femenino, que presentó una fractura patológica de fémur izquierdo traumática, en la cual se diagnosticó una displasia fibrosa poliostótica. Por presentarse acompañada de pubertad precoz periférica configura el denominado síndrome de McCune-Albright. El control y tratamiento fue multidisciplinario. El equipo de traumatología realizó osteosíntesis con placa y tornillos de la lesión ósea con evolución a la consolidación en plazos habituales (tres meses). A los seis meses de seguimiento la niña se encuentra sin dolor y sin repercusiones funcionales para la vida diaria. Del punto de vista endocrinológico se realizó tratamiento de su pubertad precoz con inhibidores de la aromatasa con el fin de mejorar su talla final y evitar repercusiones psicológicas y emocionales.

En este estudio se analizan características de esta patología y su pronóstico vital y funcional.

Palabras clave: Displasia fibrosa poliostótica

Síndrome de McCune-Albright

Pubertad precoz periférica

Key words: $\quad$ Polyostotic fibrous dysplasia

McCune-Albright syndrome

Peripheral precocious puberty

1. Médico residente, Clínica Traumatología y Ortopedia CHPR.

2. Profesor adjunto, Clínica Traumatología y Ortopedia CHPR.

3. Médico residente, Clínica de Endocrinología y Metabolismo, Universidad de la República.

4. Profesora de la Clínica de Endocrinología y Metabolismo, Universidad de la República.

Clínica de Traumatología y Ortopedia Pediátrica.

Clínica de Endocrinología y Metabolismo, Facultad de Medicina, Universidad de la República, Uruguay.

Correspondencia: Dr. Tomás Rienzi. Correo electrónico: rienzitomas@gmail.com

Los autores declaran no tener conflictos de intereses.

Recibido: $14 / 2 / 20$

Aprobado: $21 / 10 / 20$

Attribution-NonCommercial 4.0 International (CC BY-NC 4.0) 


\section{Introducción}

La displasia fibrosa (DF) es una lesión ósea benigna de baja frecuencia, congénita pero no hereditaria, debida a la mutación somática en el embrión de una célula madre y caracterizada por la proliferación medular de un tejido fibroso que encierra una osteogénesis inmadura. En ocasiones causa dolores, fracturas y deformaciones óseas ${ }^{(1)}$. Fue descrita por primera vez por Lichtenstein en 1938 y por Lichtenstein y Jaffe en $1942^{(1)}$. Se puede presentar en cualquier hueso del esqueleto. Esta lesión osteofibrosa puede afectar un hueso (monostótica) o varios (poliostótica), siendo la forma monostótica de seis a diez veces más frecuente ${ }^{(2)}$. Se han descrito complicaciones más severas para el caso de las formas poliostóticas, obligando además a descartar las formas sindromáticas como el McCune-Albright, síndrome de Mazabraud y la displasia osteofibrosa.

El síndrome de McCune-Albright (SMA) es de muy baja frecuencia, en Uruguay no contamos con datos estadísticos. En Francia se describe una incidencia estimada de 1/100.000-1/1.000.000 habitantes ${ }^{(3)}$. Este síndrome se conforma por la asociación de DF poliostótica y una o más manifestaciones extraesqueléticas: lesiones hiperpigmentadas en piel (manchas color café con leche) y endocrinopatías ${ }^{(4)}$. De estas últimas la más frecuente es la pubertad precoz, principalmente en niñas.

El SMA resulta de mutaciones esporádicas somáticas poscigóticas en el gen que codifica la subunidad á de la proteÍna Gs (GNAS1). Esta proteÍna actúa en la transducción de senbales mediante la unión a la adenil-ciclasa productora de adenosín monofosfato cÍclico (AMPc). El AMPc intracelular normalmente estimula la proliferación de las células en las glándulas (tiroides, corteza suprarrenal, ovario y pituitaria), que dan lugar a los tumores hiperfuncionantes en estos pacientes. La patogénesis de las lesiones óseas no es clara, se cree que la proteĺna Gs activada por mutaciones puede ejercer su efecto patogénico sobre los osteoclastos o fibroblastos llevando a la DF.

Es fundamental el diagnóstico oportuno y temprano de dicho síndrome para lograr un adecuado tratamiento y evitar complicaciones a corto y largo plazo. Se presenta en este estudio el caso de una niña de 8 años con DF poliostótica asociada a alteraciones endócrinas, la cual corresponde a un SMA.

\section{Caso clínico}

Escolar, sexo femenino, de 8 años, que se presenta en el servicio de traumatología con una fractura patológica de fémur izquierdo. Fractura secundaria a una caída desde su altura, mientras patinaba, quedando con dolor e impotencia funcional absoluta en miembro inferior iz-

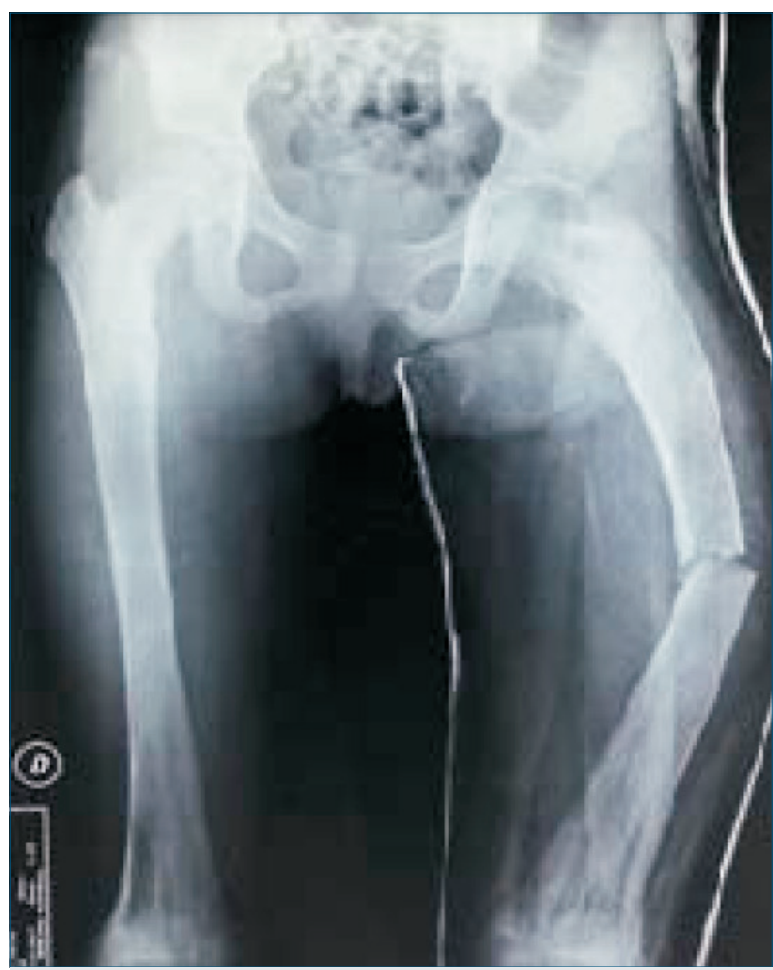

Figura 1. Displasia fibrosa en ambos fémures, con la fractura que motivó la consulta. Podemos observar la deformación de fémur proximal izquierdo. Esta deformidad en varo también es denominada en cayado de pastor (shepherd's crook) por su similitud al mango del pastor de ovejas (izquierda).

quierdo. Relató como único antecedente del polo óseo una cojera leve sin estudio.

Del balance lesional, presentó un síndrome fracturario de fémur izquierdo, mostró piel sana y el examen neurovascular fue normal. En la radiografía, presentó una fractura de fémur izquierdo medio diafisaria que asienta en un hueso de características patológicas (figura 1).

Se evidencia un trazo de fractura transversal, medio diafisaria, desplazada, en un hueso con alteraciones en su estructura dado por imágenes radiolúcidas endomedulares que adelgazan la cortical. A su vez, se acompaña con la deformación en varo de la extremidad proximal de fémur en cayado de pastor. Estas alteraciones morfológicas nos orientan hacia a una DF. Debido a que asienta en un hueso con elementos sugestivos de un tumor benigno subyacente por presentar bordes bien delimitados, sin reacción perióstica, sin compromiso de partes blandas, que además se destaca por localizaciones múltiples, nos orientamos a DF y fibroma no osificante a confirmar por anatomía patológica. Las radiografías iniciales mostraron lesiones radiolúcidas con bordes escleróticos en diáfisis de ambos húmeros, a lo largo del fémur, la tibia y 


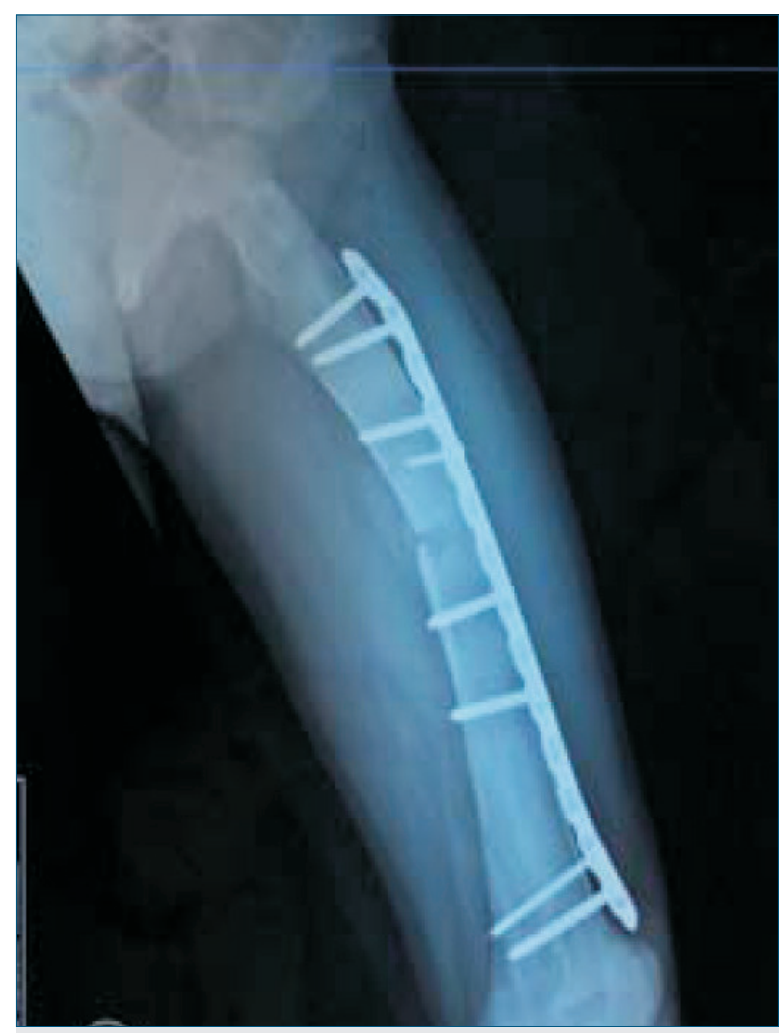

Figura 2. Control posoperatorio inmediato donde se observa una buena reducción de la fractura.

peroné izquierdos, así como el fémur y tibia derechos. Se destaca, a su vez, cartílagos de crecimiento abiertos en ambos húmeros, tibias y fémures.

La forma más rápida de determinar la distribución de la lesión en esqueleto es la gammagrafía ósea.

Este estudio evidenció lesiones poliostóticas a predominio de hemicuerpo izquierdo, como también en cráneo en región témporo-frontal, occipital izquierdo, ilíaco izquierdo, sexto y noveno arcos costales izquierdos y huesos de pie izquierdo. Las imágenes de SPECT mostraron hipercaptación en cuerpo de L3 (figura 2).

Con el planteo diagnóstico clínico-imagenológico de fractura de fémur izquierdo patológica, se realiza osteosíntesis mediante reducción abierta y fijación interna con placa bloqueada larga, al tiempo que se realiza biopsia de fragmentos óseos para el diagnóstico anatomopatológico (figuras 3 y 4). El resultado de anatomía patológica informa proliferación osteofibrosa constituida por células ahusadas que se disponen en un patrón fascicular, conformación de trabéculas óseas inmaduras de forma irregular sin ribete osteoblástico focal. En suma: DF.

En este contexto se realizó diagnóstico clínico, imagenológico, anatomopatológico de DF poliostótica.

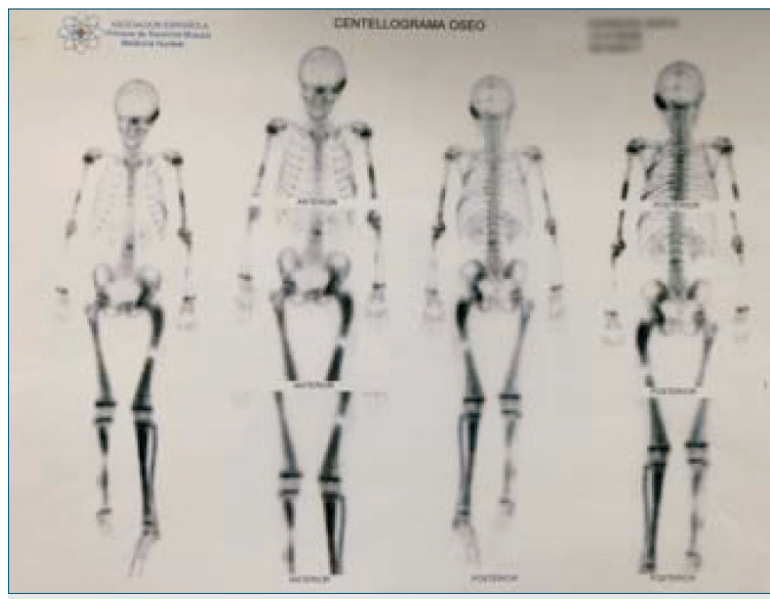

Figura 3. Centellograma óseo que evidencia lesiones poliostóticas a predominio del hemicuerpo izquierdo. Otras lesiones, además de las ya mencionadas. Las imágenes mostraron hipercaptación del radiotrazador en cráneo en región témporo-frontal occipital izquierdo e ilíaco izquierdo.

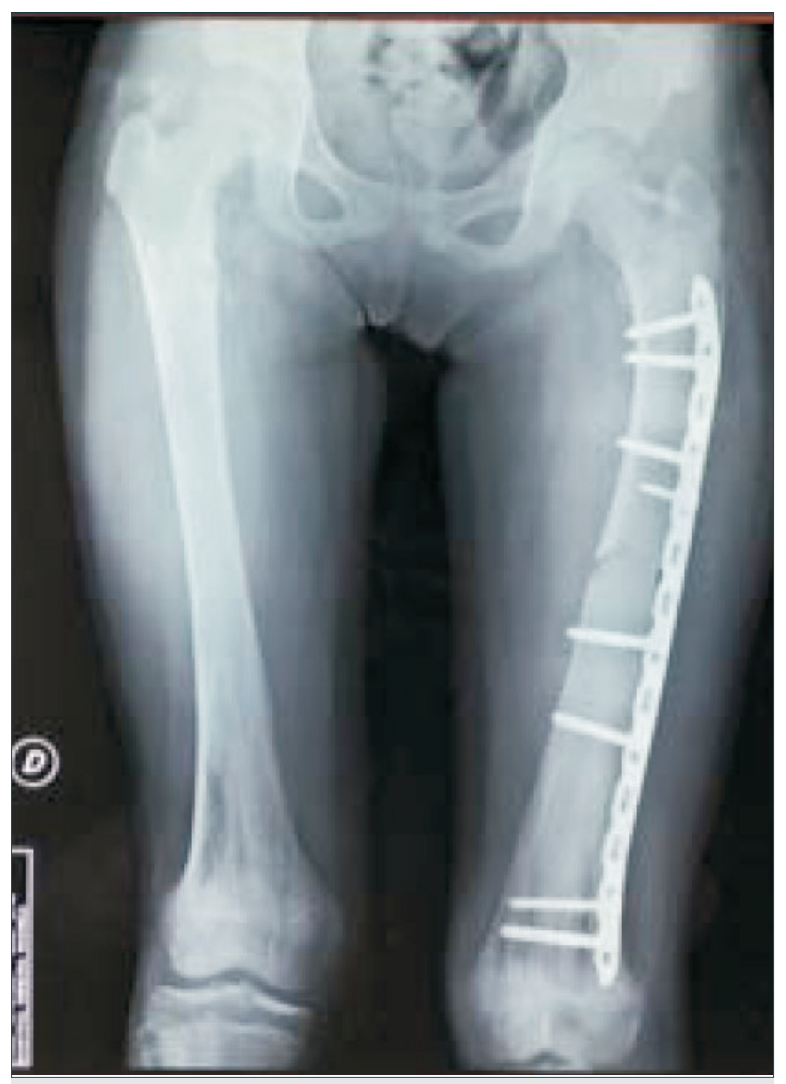

Figura 4. Radiografía control tres meses posoperatorio donde se visualiza consolidación ósea. Se presenta sin dolor y se indica apoyo total, luego de haber realizado su recuperación con muletas y apoyo parcial progresivo. 


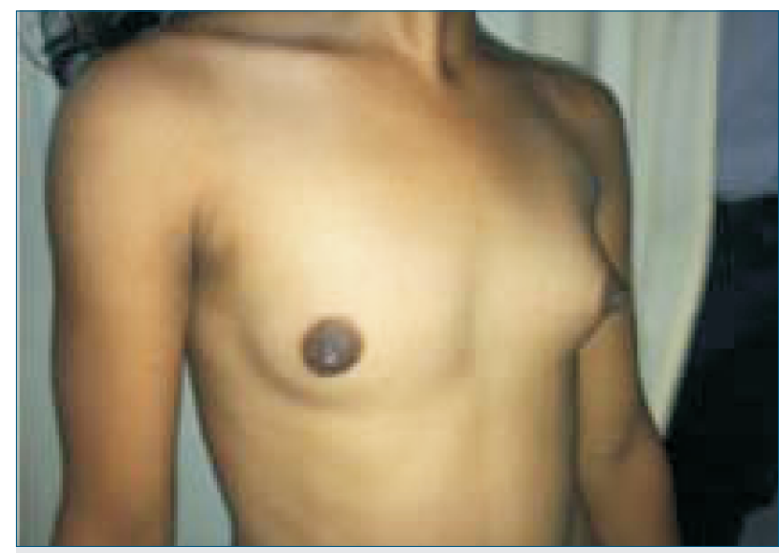

Figura 5. Mamas Tanner III: glándula mamaria que sobresale la areola, sin sobreelevación del pezón, con este último hiperpigmentado ${ }^{(5)}$

Del punto de vista endocrinológico la paciente presentó su menarquia a los 6 años de edad (no cíclica), luego agregó telarquia y por último la pubarquia.

Se encontró al examen físico, antropometría: talla de $135 \mathrm{~cm}$ (Z score: $+0,75)$, peso de $31 \mathrm{~kg}$ con un índice de masa corporal (IMC) de 17 ( $Z$ score: $+0,68)$ y una velocidad de crecimiento $(\mathrm{Vc})$ calculada de $10 \mathrm{~cm} /$ año $(>\mathrm{p} 97$ para edad y sexo). A nivel de piel, no presentó lesiones hiperpigmentadas. Mamas Tanner III bilateralmente y vello púbico Tanner II, basado en la clasificación de Tanner y colaboradores en 1962 (figura 5).

La edad ósea correspondió a 12 años, para una edad cronológica de 8 , lo cual se considera adelantada (>2 desvíos estándar para edad y sexo), basado en el Radiographic Atlas of Scheletal Development of the hand and wrist de W. W. Greulich y S. I. Pyle.

De la bioquímica se destaca el perfil hormonal gonadal con valores disminuidos de la hormona folículo estimulante (FSH) y hormona luteinizante (LH) (realizado con método ICMA), con asociación de estradiol elevado (tabla 1). La dosificación de la hormona tirotropina (TSH), tetrayodotiroxina (T4), prolactina y el factor de crecimiento insulínico tipo 1 (IGF1) se encontraban dentro del rango de normalidad para la edad y el sexo.

La ecografía ginecológica informó útero AVF 53 por $28 \mathrm{~mm}$, endometrio $6 \mathrm{~mm}$. Ovario derecho volumen de $2 \mathrm{cc}$, ovario izquierdo volumen de $2,4 \mathrm{cc}$ con dos folículos de 16 y $17 \mathrm{~mm}$.

Dada la presentación clínica y paraclínica:

- Pubertad precoz determinada por la aparición de caracteres sexuales secundarios a los 6 años, acompañada de:

- Edad ósea adelantada y Vc acelerada.

- Valores hormonales en sangre con estradiol alto y LH, FSH disminuidos.
Tabla 1. Perfil hormonal gonadal.

\begin{tabular}{lcc}
\hline \multicolumn{1}{c}{ Paraclínica } & Resultados & Normal \\
\hline Estradiol & $434 \mathrm{pg} / \mathrm{ml}$ & $<20 \mathrm{pg} / \mathrm{ml}$ (prepuberales) \\
FSH & $<0,1$ & Niñas 6 a 10 años $0,2-2,7 \mathrm{mUI} / \mathrm{mL}$ \\
LH & $<0,1$ & $<12 \mathrm{mUl} / \mathrm{mL}$ \\
\hline & & \\
\hline
\end{tabular}

- Ecografía ginecológica con características puberales y quistes ováricos mayores a $10 \mathrm{~mm}$.

Corresponde a una pubertad precoz periférica, la cual asociada a la DF poliostótica, es típico del SMA.

En lo que respecta al tratamiento endocrinológico se inició letrozole $1,5 \mathrm{mg} / \mathrm{m}^{2} / \mathrm{día}^{(* *)}$.

En sexta y novena, arcos costales izquierdos y huesos de pie izquierdo. Las imágenes de SPECT mostraron hipercaptación en cuerpo de L3. Figura 5. Mamas Tanner III: glándula mamaria que sobresale la areola, sin sobreelevación del pezón, con este último hiperpigmenta$\mathrm{do}^{(5)}$.

\section{Discusión}

El SMA fue descrito originalmente en 1937 por la tríada clásica de alteraciones de la piel (manchas color café), pubertad precoz y DF poliostótica unilateral ${ }^{(4,6)}$. Luego se describieron otras patologías asociadas: hipertiroidismo, compromiso hepático, cardíaco, exceso de hormona de crecimiento y de cortisol.

Debido a la mutación del gen GNAS1, fisiopatológicamente a nivel del ectodermo afecta sobre todo a los melanocitos de la piel, en el mesodermo afecta el hueso causando alteraciones en precursores osteoblásticos y en el endodermo afecta las glándulas endócrinas, como gónadas, tiroides, hipófisis, suprarrenales, etcétera ${ }^{(7,8)}$. Su diagnóstico se realiza debido a la asociación de dos elementos de la tríada clásica y se confirma mediante anatomía patológica o mediante toma de ADN en sangre, piel o tejido afectado.

$\mathrm{La} \mathrm{DF}$ es una lesión ósea benigna, poco frecuente. Se desconoce su verdadera incidencia, dado que existe un porcentaje considerable que son asintomáticos. A pesar de ello, se ha reportado que se encuentra entre el 5\% y $7 \%$ de los tumores óseos benignos ${ }^{(2)}$. Dentro de estas lesiones, $50 \%-70 \%$ son monostóticas, $20 \%-30 \%$ son poliostóticas y $3 \%-10 \%$ presentan un SMA, revelando una muy baja frecuencia en esta patología.

Existe solo 1\% de riesgo de malignidad, el cual se ha visto asociado a tratamientos previos con radioterapia, lo que obliga a un seguimiento más cercano de estos pa- 
cientes $^{(9)}$. El pronóstico en el caso de malignización es pobre.

Las manifestaciones extraesqueléticas de DF dentro del SMA aparecen en los 3 a 10 años de edad ${ }^{(10)}$, que en su mayoría presentan clínica a los 5 años. En esta niña la primera manifestación extraesquelética fue su menarquia a los 6 años, por la cual no consulta médico, con progresión de su pubertad precoz.

La pubertad precoz corresponde a un pilar diagnóstico del SMA ${ }^{(11)}$, y se define como la presencia de caracteres sexuales secundarios en la niña antes de los 8 años, como se presentó en la paciente. La niña tenía todas las características que se describen en una pubertad de origen periférica (PPP), la cual se produce como resultado de la producción de estrógenos gonadotropina independiente, autonómica de los ovarios debido a la mutación descrita. Se diferencia de la central (PPC) por presentar primero la menarquia (no cíclica) y luego el resto de caracteres sexuales secundarios, ni ordenada, ni progresiva. Debido al aumento de estrógenos en sangre la velocidad de crecimiento se acelera (> p95 para edad y sexo) y la edad ósea suele estar adelantada. Bioquímicamente la PPP se presenta con valores de estradiol elevado en plasma y supresión de gonadotrofinas. Por último, la ecografía ginecológica puede dar un aumento del volumen de ovario unilateral y la presencia de quistes foliculares mayores a $10 \mathrm{~mm}$. Todo esto se expresó en la paciente con un diagnóstico tardío.

Las manchas color café con leche se presentan en dos tercios de los SMA y hasta en un 50\% de las DF poliostóticas. Corresponden al tercer pilar diagnóstico del SMA. Pueden ser una pista temprana para el diagnóstico, siendo típicamente la primera manifestación clínica. En el caso de nuestra paciente, no se encuentran dichas lesiones, pero no excluye el diagnóstico de SMA. Se caracterizan por presentar a menudo un tamaño mayor a 2 $\mathrm{cm}$ de bordes irregulares, recortados (tipo costa de Maine) y, por lo general, respetan la línea media.

En cuanto a otras manifestaciones extraesqueléticas de la enfermedad, se describe afectación de la glándula tiroides en dos tercios de los $\operatorname{casos}^{(12)}$, más frecuentemente hipertiroidismo. Se debe dosificar en sangre TSH y realizar una ecografía para descartar alteraciones estructurales. Solo la mitad de los pacientes que presentan alteraciones en la ecografía tienen un franco hipertiroidismo clínico. Es importante su diagnóstico, ya que sus alteraciones pueden avanzar la edad ósea.

Los tejidos con DF producen FGF23, lo cual lleva a la pérdida de fosfato renal, bajando el fósforo en sangre. La hipofosfaturia se relaciona con mayor índice de fractura a menor edad y además mayor dolor óseo. Su tratamiento se realiza con fosfato y vitamina $\mathrm{D}^{(4,13)}$, lo cual resulta en una mejora de sus síntomas. Por último, hay que descartar hiperproducción de hormona de crecimiento como, a su vez, de la prolactina, que se pueden ver en estos pacientes asociadas a tumores hipofi$\operatorname{sarios}^{(13)}$.

En cuanto a las complicaciones óseas la fractura es frecuente y con gran índice de recurrencia, lo que repercute directamente en la calidad de vida del paciente y representa un desafío para el cirujano ortopédico en equipo multidisciplinario con endocrinología.

En cuanto a la fractura de fémur que presenta nuestra paciente, podemos decir que asienta en un hueso con alteración en su estructura y morfología. El reemplazo de hueso medular por tejido fibroso conduce a una pérdida del patrón trabecular, confiriendo en la radiografía la lesión un aspecto en vidrio esmerilado.

La deformación en cayado de pastor es característica de la DF poliostótica.

En un análisis de 46 casos, entre 1994 y $2010^{(14)}$, se vio que existen seis tipos de deformidades femorales en pacientes con DF poliostótica, del tipo I al VI más severo. El diagnóstico suele ocurrir a los 5 años de edad. Dentro de las conclusiones, se vio que la deformación avanza a la peoría al transcurrir el tiempo. Nuestra paciente se encuentra en el tipo IV, donde se observa afectación de todo el fémur, lo cual tiene implicancias clínicas importantes, deformidad de muslo, cojera y dolor. Las fracturas de fémur suelen ocurrir complicando la situación clínica. Más allá del tratamiento quirúrgico es esperable que su deformación continúe avanzando.

En un estudio realizado por Leet A y colaboradores, publicado en $2004^{(15)}$, con 35 pacientes, la incidencia de fractura de DF poliostótica mostró un pico entre los 6 a 10 años, que disminuye en cuanto aumenta la edad. Este estudio solo toma en cuenta las fracturas en los miembros. El sitio de mayor fractura es el fémur, seguido por húmero, tibia y antebrazo. En este estudio de 35 pacientes, 19 asociaban pubertad precoz, 9 hipertiroidismo y 7 otras endocrinopatías. Además, se encontró que 12 pacientes presentaban una fosfaturia elevada. El estudio concluye que los pacientes que presentan anormalidades metabólicas tienen fracturas precozmente. Se vio una incidencia más temprana y un aumento en la frecuencia en pacientes con fosfaturia elevada.

La consolidación ósea suele ser normal. La decisión de tratamiento dependerá del tipo de fractura, localización y deformidad ósea.

Las repercusiones endócrinas secundarias a la pubertad precoz tienen como consecuencia implicancias psicosociales, emocionales y disminución de la talla final (en algunos casos talla baja) por cierre precoz de los cartílagos de crecimiento, dado el aumento de los niveles de estrógenos en sangre. Se han planteado otros potenciales riesgos de la adultez: tendencia a la obesidad, 
aumentos de cifras de la presión arterial, alteración del metabolismo de hidratos de carbono y aumento del riesgo cardiovascular con incremento de la morbimortalidad, por lo que es un reto diagnóstico y es fundamental el tratamiento oportuno para el médico tratante.

Ensayos clínicos no controlados encontraron que los bifosfonatos disminuyen el dolor, mejoran la apariencia de las lesiones en las radiografías y disminuyen la incidencia de fractura, mejorando la calidad de vida ${ }^{(16,17)}$. Se sugiere también que la combinación de bifosfonatos e instrumentación quirúrgica puede ser beneficiosa. El uso de bifosfonatos vía intravenosa ha demostrado ser efectivo en lesiones óseas dolorosas; el uso de pamidronato parenteral ha sido reportado como que logra mejoría de la densidad ósea en forma significativa ${ }^{(18)}$.

Desde el punto de vista ortopédico el tratamiento de las lesiones óseas puede ser un desafío, en particular en el caso de lesiones múltiples o deformidades mayores. Sabemos que en el caso de lesiones monostóticas el tratamiento es principalmente ortopédico y solo está indicada la cirugía en caso de una gran deformidad y de un gran dolor. El tratamiento quirúrgico profiláctico con enclavijado solo es recomendado en la DF infantil. Por lo general, el tratamiento quirúrgico se indica frente a fracturas patológicas recurrentes, cuando las lesiones causan deformidad progresiva y asocian dolor persistente. Las fracturas patológicas mínimamente desplazadas consolidan en un plazo normal, por lo que el retraso de consolidación o pseudoartrosis no es un problema en estos pacientes. La principal complicación es la deformación progresiva.

Frente a una deformidad ósea el objetivo del tratamiento es realinear, particularmente en los huesos de carga. Nuestra paciente presenta una cojera debida a la desviación en varo de su cadera, por la cual está planificada su corrección quirúrgica. En niños pequeños se prefiere realizar el tratamiento ortopédico, pero en caso de fracturas repetidas en huesos largos o compromiso del fémur proximal, se prefiere el tratamiento quirúrgico.

La decisión de la técnica quirúrgica se toma con los mismos principios de osteosíntesis que en huesos no patológicos, teniendo en cuenta las alteraciones en cada caso individualizado.

El enclavijado puede ser un desafío por las deformaciones y por la calidad de hueso.

Se espera aun mayor dificultad en un esqueleto maduro. En la cirugía no se han visto beneficios al realizar curetaje, ya que éste lleva por lo general a la recurrencia local. No se recomienda utilizar injerto óseo, pues se ha observado que éste se reabsorbe y es sustituido por fibrosis nuevamente.

Leet colaboradores ${ }^{(19)}$. Se han visto mejores resultados con injerto óseo vascularizado ${ }^{(20)}$.
La decisión óptima del tratamiento quirúrgico no ha sido determinada. Hay quienes recomiendan enclavijado endomedular en lesiones en huesos de carga con DF severa porque provee estabilidad por mayor tiempo, previene nuevas fracturas y el aumento de la deformidad. El enclavijado, como contrapartida, puede lesionar la fisis y ser de difícil colocación dependiendo de la deformidad.

La decisión en nuestra paciente fue una placa bloqueada, la cual fue moldeada respetando la fisis. Se lograron los objetivos de realineación y consolidación en los tiempos apropiados (figura 3 ). Se va a requerir un seguimiento cercano de esta paciente, ya que sabemos que la osteosíntesis soluciona el problema agudo de la fractura, pero no la enfermedad de base.

En la evolución, osteotomías pueden ser necesarias en caso de consolidación viciosa o si está presente la deformación en fémur proximal en cayado de pastor, como en el caso de nuestra paciente, que presenta una leve cojera que se espera evolucione a la peoría en el caso de no tratarla.

Por último, en lo que respecta al tratamiento de la pubertad precoz periférica, es inicialmente el seguimiento clínico. De ser progresivo, como es el caso de la niña analizada, se usan fármacos inhibidores de la aromatasa con acción antiestrogénica (letrozol) ${ }^{(21)}$. Estas niñas en la adultez pueden tener comprometida la fertilidad. La descendencia no tiene por qué verse afectada.

\section{Conclusión}

Estamos frente a una niña de 8 años que presenta SMA. Es fundamental el diagnóstico temprano y el adecuado abordaje multidisciplinario. El diagnóstico de su enfermedad fue a partir de una fractura patológica de fémur izquierdo. Desde el punto de vista traumatológico, la niña es tratada mediante osteosíntesis de fémur con placa bloqueada lateral. Presenta una buena evolución clínica imagenológica con un buen proceso de consolidación ósea en los tiempos esperados, sin dolor, y vuelta a la actividad diaria. Su pronóstico funcional es bueno, siendo probablemente necesaria una nueva intervención a futuro debido a la deformación en varo de su cadera. Se restringe la actividad deportiva y se actúa en la prevención de nuevas fracturas. La paciente se encuentra en control y tratamiento con endocrinólogo por su pubertad precoz, seguimiento de repercusiones en el metabolismo fosfo-cálcico y posible aparición de otras patologías endócrinas.

\section{Summary}

Introduction: polyostotic fibrous dysplasia is a low prevalence disease, and for this reason, it often goes undetected. When associated to endocrinopathies and/or 
hyperpigmented skin lesions we speak about McCune Albright syndrome.

Clinical case: eight-year old school girl who presented pathological fracture of the left femur, which was diagnosed as polyostotic fibrous dysplasia. As it was accompanied by peripheral precocious puberty it constituted an indicative clinical picture of the so-called $\mathrm{McCu}$ ne Albright. Control and treatment were multidisciplinary. The traumatology team performed osteosynthesis with plaques and nails to fix the bone lesion, and evolution consolidated in a regular time frame (3 months). Upon six months follow-up, the girl has no pain and presents no functional repercussion in daily life. From the endocrinological perspective, the girl received precocious puberty treatment with aromatase inhibitors with the purpose of improving her final size and avoid psychological and emotional implications.

The study presents the characteristics of this condition, as well as its vital and functional prognosis

\section{Resumo}

Introdução: a displasia fibrosa poliostótica é uma doença de prevalência muito baixa, por isso seu diagnóstico passa despercebido na maioria dos casos. Quando associada a endocrinopatias e / ou lesões cutâneas hiperpigmentadas, corresponde à síndrome de McCune Albright.

Caso clínico: estudante do sexo feminino, 8 anos, com quadro de fratura patológica traumática do fêmur esquerdo, com diagnóstico de displasia fibrosa poliostótica. Por estar acompanhada de puberdade precoce periférica, configura a chamada síndrome de McCune Albright. O controle e o tratamento foram multidisciplinares. A equipe de trauma realizou osteossíntese de placa e parafuso da lesão óssea com progressão à consolidação nos prazos usuais (3 meses). Aos 6 meses de seguimento, a paciente não apresenta dor e tampouco repercussões funcionais no dia a dia. Do ponto de vista endocrinológico, sua puberdade precoce foi tratada com inibidores da aromatase para melhorar sua altura final e evitar repercussões psicológicas e emocionais.

Este estudo analisa as características desta patologia, seu prognóstico vital e funcional.

\section{Bibliografía}

1. Chapurlat R, Meunier P. Fibrous dysplasia of bone. Baillieres Best Pract Res Clin Rheumatol 2000; 14(2):385-98. doi: 10.1053/berh.1999.0071

2. Santini-Araujo E, Kalil R, Bertoni F, Park Y. Tumors and tumor-like lesions of bone. Basel: Springer Nature, 2020.

3. Chapurlat R, Orcel P. Fibrous dysplasia of bone and $\mathrm{McCu}-$ ne-Albright syndrome. Best Pract Res Clin Rheumatol 2008; 22(1):55-69. doi:10.1016/j.berh.2007.11.004
4. Dumitrescu C, Collins M. McCune-Albright syndrome. Orphanet J Rare Dis 2008; 3:12. doi: 10.1186/ 1750-1172-3-12

5. Temboury Molina M. Desarrollo puberal normal: pubertad precoz. Rev Pediatr Aten Primaria 2009; 11(Supl 16):127-42.

6. Boyce A, Chong W, Yao J, Gafni R, Kelly M, Chamberlain C, et al. Denosumab treatment for fibrous dysplasia. J Bone Miner Res 2012; 27(7):1462-70. doi: 10.1002/j bmr.1603

7. Lovy A, Dowdell J, Keswani A, Koehler S, Kim J, Weinfeld S, et al. Nonoperative Versus Operative Treatment of Displaced Ankle Fractures in Diabetics. Foot Ankle Int 2017; 38(3):255-60. doi: 10.1177/1071100716678796

8. Weinstein LS. Activating mutations of the stimulatory G protein in the McCune- Albright syndrome. N Engl J Med 1991;325:1688-95.

9. Ruggieri P, Sim F, Bond J, Unni K. Malignancies in fibrous dysplasia. Cancer 1994; 73(5):1411-24.

10. Collins M, Singer F, Eugster E. McCune-Albright syndrome and the extraskeletal manifestations of fibrous dysplasia. Orphanet J Rare Dis 2012; 7(Suppl 1):S4. doi: 10.1186/ 1750-1172-7-S1-S4

11. Schwindinger W, Francomano C, Levine M. Identification of a mutation in the gene encoding the alpha subunit of the stimulatory $\mathrm{G}$ protein of adenylyl cyclase in McCune-Albright syndrome. Proc Natl Acad Sci U S A 1992; 89(11):5152-6. doi: 10.1073/pnas.89.11.5152

12. Celi F, Coppotelli G, Chidakel A, Kelly M, Brillante B, Shawker T, et al. The role of type 1 and type $25^{\prime}$-deiodinase in the pathophysiology of the 3,5,3'-triiodothyronine toxicosis of McCune-Albright syndrome. J Clin Endocrinol Metab 2008; 93(6):2383-9. doi: 10.1210/jc.2007-2237

13. Carpenter T, Imel E, Holm I, Jan de Beur S, Insogna K. A clinician's guide to X-linked hypophosphatemia. J Bone Miner Res 2011; 26(7):1381-8. doi: 10.1002/jbmr.340

14. Ippolito E, Valentini M, Lala R, De Maio F, Sorge R, Farsetti P. Changing pattern of femoral deformity during growth in polyostotic fibrous dysplasia of the bone: an analysis of 46 cases. J Pediatr Orthop 2016; 36(5):488-93. doi: 10.1097/ BPO.0000000000000473

15. Leet A, Chebli C, Kushner H, Chen C, Kelly M, Brillante B, et al. Fracture incidence in polyostotic fibrous dysplasia and the McCune-Albright syndrome. J Bone Miner Res 2004; 19(4):571-7. doi: 10.1359/JBMR.0301262

16. Chapurlat R, Delmas P, Liens D, Meunier P. Long-term effects of intravenous pamidronate in fibrous dysplasia of bone. J Bone Miner Res 1997; 12(10):1746-52. doi: 10.1359/jbmr.1997.12.10.1746

17. Lala R, Matarazzo P, Bertelloni S, Buzi F, Rigon F, de Sanctis C. Pamidronate treatment of bone fibrous dysplasia in nine children with McCune-Albright syndrome. Acta Paediatr 2000; 89(2):188-93. doi: 10.1080/080352500750028816

18. Isaia G, Lala R, Defilippi C, Matarazzo P, Andreo M, Roggia $\mathbf{C}$, et al. Bone turnover in children and adolescents 
with McCune-Albright syndrome treated with pamidronate for bone fibrous dysplasia. Calcif Tissue Int 2002; 71(2):121-8. doi: 10.1007/s00223-001-1098-7

19. Leet A, Boyce A, Ibrahim K, Wientroub S, Kushner H, Collins M. Bone-Grafting in Polyostotic Fibrous Dysplasia. J Bone Joint Surg Am 2016; 98(3):211-9. doi: 10.2106/JBJS.O.00547

20. Perea-Tortosa D, García-López A, Saura-Sánchez E, Aguirre-Pastor A. Treatment of fibrous dysplasia of the pro- ximal femur by means of pedicled iliac crest bone graft: a case report. Microsurgery 2011; 31(1):56-8. doi: 10.1002/micr. 20830

21. Feuillan P, Calis K, Hill S, Shawker T, Robey P, Collins M. Letrozole treatment of precocious puberty in girls with the McCune-Albright syndrome: a pilot study. J Clin Endocrinol Metab 2007; 92(6):2100-6. doi: 10.1210/jc.2006-2350

\section{Contribución de autores}

Tomás Rienzi, https://orcid.org/0000-0001-5806-8727. Concepción, diseño, ejecución, análisis, interpretación de los resultados, redacción, revisión crítica.

Claudio Silveri, https://orcid.org/0000-0002-2607-7749. Concepción, diseño, interpretación de los resultados, redacción, revisión crítica. Beatriz Mendoza, https://orcid.org/0000-0002-7898-9336. Concepción, diseño, interpretación de los resultados, redacción, revisión crítica.

Gottardo Bianchi, https://orcid.org/0000-0001-8222-4435. Concepción, diseño, interpretación de los resultados, redacción, revisión crítica. 